\title{
A STUDY OF GOOGLE CLOUD SERVICES
}

\author{
Sushama Pawar \\ Vidyaankar Poytechnic \\ Mumbai, Maharashtra, India
}

\author{
Chetashri bhusari \\ Vidyaankar Poytechnic \\ Mumbai, Maharashtra, India
}

\author{
Archana Gopnarayan \\ Vidyaankar Poytechnic \\ Mumbai, Maharashtra, India
}

\begin{abstract}
One of the leaders of cloud APIs is Google Cloud Platform (GCP) today. While it was founded just few years ago, due to its suite of public cloud services built on a massive, strong infrastructure, GCP has achieved substantial expansion. By accessing GCP, GCP enables developers to use these resources. This study gives us an insight into the global infrastructure of Google. They also offer insight into the topic of Regions, zones and initiatives that form the environment of the GCP. This research paper focuses on cloud computing, a summary of the various cloud computing services provided by GCP, Understanding the GCP environment, Different Compute, Storage, Big Data and AI tools offered by GCP, Google Network Service Offerings.
\end{abstract}

Keywords: Google Cloud platform, GCP environment, big data, AI tool, storage services, server less computing.

\section{INTRODUCTION}

Over the past few years, cloud computing has been The emerging area in the IT market. Numerous providers of cloud services Competing computing, storage, networking, and Web hosting facilities thus offering coverage several continents are offering the highest on-demand rates and Achievement. These services have a heterogeneous name [1], Characteristics and features. In addition, there are cloud providers

Depend on technically different Application Programming Interfaces (APIs), i.e. cloud management interfaces that offer a solution to your needs. Remote programmatic access to their infrastructure. As for the semantics of these cloud APIs, they are only specified informally, i.e. defined in natural language on the website of the API. The creator may not have known the exact actions of the project.

Google Cloud Platform (GCP), provided by Google, is a suite of cloud computing services that operate on the same infrastructure that Google uses internally for its end-user products, such as Google Search, Gmail, file storage, and YouTube. In addition to a collection of management tools, it offers a variety of modular cloud services, including computation, data storage, data analytic $\mathrm{s}$ and machine learning. Registration includes the information of a credit card or bank account. Google Cloud Platform offers infrastructure as a service, a platform as a service, and a server less computing environment.

In April 2008, Google unveiled App Engine, a platform for creating and storing web applications in Google operated data centers, the company's first cloud computing service. The service was generally accessible in November 2011. Google has added several cloud services to the platform since the App Engine was released. Google Cloud Platform is part of Google Cloud, and comprises the public cloud infrastructure of the Google Cloud Platform as well as Google Workspace (formerly G Suite), business versions of Android and Chrome OS, and application programming interfaces (APIs) for machine learning and enterprise mapping services.

\section{GOOGLE CLOUD RESOURCES}

Google Cloud consists of a set of physical assets contained in Google data centers across the globe, such as computers and hard disc drives, and virtual resources, such as virtual machines ( VMs). Each location for a data centre is in a area. Asia, Australia, Europe, North America, and South America have regions open. Each area is a series of zones which are separated within the region from each other. A name that combines a letter identifier with the name of the area is identified by each zone. For example, zone an is called asia-east1-a in the East Asia region.

There are many advantages to this allocation of resources, including flexibility in the event of failure and decreased latency by locating resources closer to customers. Some guidelines on how resources can be used together are also implemented by this distribution.

\section{GLOBAL, REGIONAL, AND ZONAL RESOURCES}

Any other resource, across regions and zones, can access some resources. Preconfigured disc files, disc screenshots, and networks are included in these global tools. Only resources that are located in the same area can access those resources. Static external IP addresses are included in these regional services. Only resources that are located in the same zone can access other resources. VM instances, their forms, and discs are included in these zonal tools. 


\section{International Journal of Engineering Applied Sciences and Technology, 2020 \\ Vol. 5, Issue 7, ISSN No. 2455-2143, Pages 227-231 \\ Published Online November 2020 in IJEAST (http://www.ijeast.com)}

The following diagram shows the relationship between global scope, regions and zones, and some of their resources:

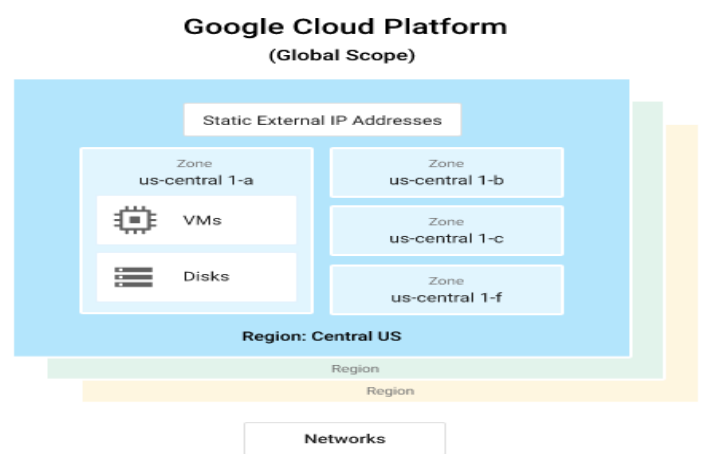

Fig.1: Google Cloud Platform

Depending on what kind of tools you are dealing with, the complexity of an operation differs. For instance, it is a global operation to build a network because a network is a global resource, while reserving an IP address is a regional operation because the address is a regional resource.

It's important to understand how these regions and zones connect when you start to customise your Google Cloud applications. For instance, you would not want to connect a disc in one region to a computer in another region, even though you could, because the lag you would add would result in poor performance.

\section{GOOGLE CLOUd SER VICES}

This summary refers to the following categories of services:

\subsection{Computing and hosting services}

Google Cloud offers you storage and hosting options. You can opt to do the following:

- Work in a server-less environment.

- Use a controlled framework platform, please.

- Container technology used to achieve a lot of versatility.

- Create your own cloud-based platform to provide the most power and versatility possible.

- You can imagine a continuum where, on the one hand, you have most of the responsibilities for handling resources and, on the other, Google has most of those responsibilities.

\subsubsection{Server less computing}

Cloud Functions, the FaaS (Functions as a Service) offering from Google Cloud, offers a server less execution environment to create and link cloud services. You write quick, single-purpose features with Cloud Functions that are attached to events emitted from your cloud infrastructure and services. When an event being observed is activated, your feature is triggered. In a fully controlled system, the code is executed. There is no need for any infrastructure to be provided or to think about handling any servers.

JavaScript, Python 3, Go, or Java can be used to write Cloud Functions. In any regular Node.js (Node.js 10), Python 3 (Python 3.7), Go (Go 1.11 or 1.13) or Java (Java 11) environment, which makes both portability and local testing a breeze, you can take your feature and run it.

For use cases that include the following, Cloud Functions are a reasonable choice:

- For scenarios like video transcoding and IoT streaming data, data processing and ETL operations.

- Web hooks to respond to the triggers of HTTP.

- Lightweight APIs that compose applications with loosely coupled logic.

- Functions of mobile backend.

\subsubsection{Application platform}

The Google Cloud platform as a service (PaaS) is the App Driver. Google does much of the resource management for you with the Software Engine. For example, if more computational resources are needed for your application because traffic to your website rises, Google scales the system automatically to provide those resources. If a security update is required for the system software, that's done for you, too.

On the App Engine, when you create your app, you can:

- Create your app and use pre-configured runtimes in Go, Java, .NET, Node.js, PHP, Python, or Ruby, or use custom runtimes to write code in any language.

- Let Google handle you with app hosting, scaling, tracking, and infrastructure.

- Link to products such as Cloud SQL, Firestore in Datastore mode, and Cloud Storage for Google Cloud storage. You can also link and host thirdparty databases such as MongoDB and Cassandra on Compute Engine, another cloud provider, onpremises, or a third-party vendor with managed Redis databases.

- To detect security vulnerabilities, use the Web Security Scanner to complement your current protected design and development processes.

\subsubsection{Virtual machines}




\section{International Journal of Engineering Applied Sciences and Technology, 2020 \\ Vol. 5, Issue 7, ISSN No. 2455-2143, Pages 227-231 \\ Published Online November 2020 in IJEAST (http://www.ijeast.com)}

The Compute Engine is Google Cloud's unmanaged computing service. Compute Engine can be considered as a service infrastructure (IaaS) because the framework offers a stable computing infrastructure, but you have to select and configure the components of the network that you want to use. It is your duty to configure, manage, and control the systems with the Compute Engine. Google can ensure that services are usable, reliable, and ready to be used by you, but it is up to you to provide and handle them. The benefit here is that you have full control and infinite versatility over the systems.

You can do the following when you build on the Compute Engine:

- To build your application, use virtual machines (VMs), named instances, just like you would if you had your own hardware infrastructure. To configure your configuration to suit your needs and your budget, you can choose from a number of example styles.

- Choose which global regions and zones your resources should be distributed in, allowing you to monitor where your data is stored and used.

- Choose which operating systems you prefer, stacks of development, languages, frameworks, services, and other software technologies.

- Create examples from public or private images.

- Using Google Cloud storage technology or any of your favourite third-party technologies.

- Using Google Cloud Marketplace to deploy preconfigured packages of software easily. For example, with just a few clicks, you can deploy a LAMP or MEAN stack.

- Build example groups to handle multiple instances together more easily.

- Use auto scaling with an instance group to automatically add and remove capacity.

- Attach and detach disks as needed.

- Use SSH to connect directly to your instances.

\subsection{Storage services}

You will likely need to store some media files, backups, or other file-like objects, regardless of your application. A range of storage services are offered by Google Cloud, including:

- In Cloud Computing, consistent, elastic, largecapacity data storage [13][14]. There are several flavours of Cloud Storage:

1. Maximum flexibility is given by Regular Cloud Storage.

2. Cloud Storage Near line is suitable for data accessed less than once a month, offering lowcost archival storage.
3. Also lower-cost archival storage is suitable for data accessed less than once a quarter via Cloud Storage Cold line.

4. The Cloud Storage Archive is the lowest cost backup and disaster recovery archival storage that is suitable for data you expect to use less than once a year.

- Compute Engine permanent discs, to be used as primary storage for your instances. Compute Engine provides both permanent hard-disk-based discs, called regular persistent discs, and persistent solid-state (SSD) discs.

- Fully-managed Filestore NFS file servers. To store data from applications running on Compute Engine VM instances or GKE clusters, you can use File store instances.

4.3 Database services

A number of SQL and NoSQL database services are offered by Google Cloud:

- A Cloud SQL SQL database that provides either MySQL or PostgreSQL databases.

- A professionally operated, mission-critical, relational Cloud Spanner database service that provides global-scale transactional consistency, schemas, SQL queries, and high-availability continuous, synchronous replication.

- Two NoSQL data storage options: Fire store, for document-like information, and Cloud Big table, for tabular information.

\subsection{Networking services}

Although App Engine handles networking for you, and GKE uses the Kubernetes model, a collection of networking services are provided by Compute Engine. These services help you load-balance resource-based traffic, create DNS records, and link your existing network to the network of Google.

\subsubsection{Networks, firewalls, and routes}

A set of networking services used by your VM instances are provided by the Virtual Private Cloud (VPC). An example may have more than one interface, but it is necessary to connect each interface to a different network. There is a default network on every VPC project. In your project, you can create additional networks, but the networks cannot be shared between projects.

The rules of firewall regulate traffic coming to instances on a network. There is a default set of firewall rules on the default network and you can build custom rules, too.

In your case, a route allows you to implement more advanced networking functions, such as creating VPNs. A route specifies how to direct packets that leave an instance. For example, a route might specify that a virtual machine 


\section{International Journal of Engineering Applied Sciences and Technology, 2020 \\ Vol. 5, Issue 7, ISSN No. 2455-2143, Pages 227-231 \\ Published Online November 2020 in IJEAST (http://www.ijeast.com)}

gateway instance that you configure and operate should handle packets destined for a specific network range.

\subsubsection{Advanced connectivity}

Google Cloud provides the following options for advanced networking if you have an existing network that you want to link to Google Cloud resources:

- Cloud Interconnect allows you, via a highly accessible, low-latency, enterprise-grade connection, to connect your current network to your VPC network. In order to link directly to Google, you can use Dedicated Interconnect or use Partner Interconnect to connect to Google through a supported service provider.

- Cloud VPN allows you to use an IPsec connection to link your current network to your VPC network. To link two Cloud VPN gateways to each other, you can also use VPN.

- Direct Peering helps you to share internet traffic at one of the broad-reaching edge network locations of Google between your business network and Google. For more information about edge positions, see Google's peering section.

- Through using service providers, Carrier Peering allows you to link your networks to Google's network edge via highly accessible, lower-latency connections. Through using a VPN tunnel between the networks, you can also expand your private network into your private Virtual Private Cloud network over Carrier Peering connections.

\subsection{Big data services}

In order to get quick answers to complex questions, big data services allow you to process and query big data in the cloud.

\subsubsection{Data analysis}

Big Query supplies services for data processing. With BigQuery, it is possible to:

- Build custom schemas which organise your information into tables and datasets.

- Using a number of sources to load data, including streaming data.

- To query large databases very easily, use SQLlike commands. BigQuery is designed for speed and optimised for it.

- Using the web UI, interface with the command line, or the API.

- Load, query, export, and use jobs to copy data.

- Data control and security through the use of permissions.

\subsubsection{Batch and streaming data processing}

Data flow includes a controlled service and a collection of SDKs that can be used for batch processing and data streaming activities. For high-volume computing, Data flow works well, especially when the processing tasks can be split into parallel workloads clearly and easily. Data flow is also great for ETL (Extract-Transform-Load) tasks that are helpful for transferring data between various storage media, translating data into a more desirable format, or loading data to a new storage device.

\subsubsection{Asynchronous messaging}

- $\quad$ Pub / Sub is a service for asynchronous messaging. Your application will submit messages to a publishing unit called a topic as JSON data structures. Since Pub / Sub topics are a global resource, other applications in projects that you own will subscribe to the topic to receive the messages in HTTP request or answer bodies. See the Pub / Sub quick start to familiarize yourself with Pub / Sub.

- The utility of Pub / Sub is not limited to big data. In many cases in which you need an asynchronous messaging service, you can use Pub / Sub. For an example that uses Pub / Sub to coordinate the Compute Engine and App Engine, see Compute Engine Efficient Task Scheduling.

\subsection{Machine learning services}

AI Platform offers a variety of powerful machine learning (ML) services. You can choose to use APIs that provide pretrained models optimized for specific applications, or build and train your own large-scale, sophisticated models using a managed Tensor Flow framework[10].

\subsubsection{Machine learning APIs}

You can use the ML of Google Cloud to build and train your own models, with different APIs. Google Cloud gives you.

- Video Intelligence API allows you to use mark identification, clear content detection, shot-change detection and regionalization technology.

- Speech-to-Text helps you to turn audio into text to support your global user base, understanding more than 110 languages and variants. The text of users dictating to an application can be transcribed, monitored and controlled by means of a speech, or audio files can be transcribed between other uses.

- Cloud Vision helps you to quickly add view recognition features, such as image marking, facial detection, OCR and clear content tagging. 


\section{International Journal of Engineering Applied Sciences and Technology, 2020 \\ Vol. 5, Issue 7, ISSN No. 2455-2143, Pages 227-231 \\ Published Online November 2020 in IJEAST (http://www.ijeast.com)}

- The Natural Language API of Cloud can be used to incorporate sentiment analysis, analysis of entities, analysis of entities, classification of content and analysis for syntax.

- Cloud Translation allows you to translate source text easily into a hundred languages supported. In situations where the root language is not identified, language identification helps.

\subsubsection{AI Platform}

The AI Platform integrates Google Cloud 's managed infrastructure with TensorFlow 's strength and versatility. You can use this to train models of your machine and host trained models to predict new cloud data.

AI Platform allows you to train models by running TensorFlow training on Google Cloud and host those models so that you can use them for new data predictions. You should focus more on your model than on hardware configurations or resource management. AI Framework handles the machine resources that your training requires to run.

\section{CONCLUSION AND ACKNOWLEDGE}

In conclusion, cloud computing is a new technical revolution that has the potential to have a significant impact on the planet. It has many advantages for its users and companies. Some of the advantages it brings to companies, for instance, are that it lowers operational expenses by investing less on maintenance and software updates and relying more on the companies themselves. Yet there are other problems that must be solved by cloud computing. We have studied many services offered by Google Cloud in our paper.

\section{REFERENCES}

[1] https://cloudacademy.com/course/designing-a-googlecloud-infrastructure

[2] https://cloud.google.com/

[3] Stephanie Challita, Faiez Zalila, Christophe Gourdin, Philippe Merle, "A Precise Model for Google Cloud Platform", Cloud Engineering (IC2E) 2018 IEEE International Conference on, pp. 177-183, 2018

[4] S. P. T., Krishnan \& Gonzalez, Jose. (2015). "Getting Started with Google Cloud Platform”. 10.1007/978-1-48421004-8_2.

[5] F. Petrillo, P. Merle, N. Moha, and Y.-G. Gu'eh'eneuc, "Towards a REST Cloud Computing Lexicon," in the 7th International Conference on Cloud Computing and Services Science (CLOSER), 2017, pp. 376-383.

[6] Wang (2012). "Enterprise cloud service architectures". Information Technology and
Management. 13 (4): $\quad$ 445-454. doi:10.1007/s10799-0120139-4. S2CID 8251298.

[7] Wang, H., He, W. \& Wang, FK. Enterprise cloud service architectures. Inf Technol Manag 13, 445-454 (2012). https://doi.org/10.1007/s10799-012-0139-4

[8] Zhao Wei; An Initial Review of Cloud Computing Services Research Development; International Conference on Multimedia Information Networking and Security; 2010. [9] Qasim Alajmi , Louay A. Al-Nuaimy, G. Jai Arul Jose , Mohammed Mastan , Mohammed A. Al-Sharafi "Cloud computing services and its Effect on tertiary education: Using Google Classroom" 2019 7th International conference on ICT \& Accessibility (ICTA) ,IEEE DOI: 10.1109/ICTA49490.2019.9144797

[10]Gabriel Beims Brascher,Rafael Weingartner, Carlos Becker Westphall "Improving cloud computing virtual machines balancing through hosts and virtual machines similarities"2017 IEEE 13th World Congress on Services [11] Jinho Seol, Seongwook Jin, Seungryoul Maeng "Secure Storage Service for IaaS Cloud Users" 2013 13th IEEE/ACM International Symposium on Cluster, Cloud, and Grid Computing

[12] Che-Pin Chang, Hsin-Ta Chiao, Yue-Shan Chang, Ching-Tsorng Tsai, Kuo-Kai Yuen1, Shyan-Ming Yuan "UCS - A Unified Cloud Storage Integration Service"2017 IEEE 7th International Symposium on Cloud and Service Computing. 